\title{
Mobile Cloud Computing In Business
}

\author{
Nilam S. Desai \\ Smt. Chandaben Mohanbhai Patel Institute of Computer Applications, Charotar \\ University of Science and Technology, Changa, Gujarat, India
}

\begin{abstract}
Cloud integrated mobile application provides the functions for building and consuming the next generation of business applications. The effect of mobile cloud computing on the traditional E-business gives the necessary solutions on the development of E-commerce businesses in cloud space and provides huge accessibility by mobile applications. Mobile application provide great accessibility for business as now a days, every person is using hand held devices for various tasks but it faces some issues. By incorporating cloud computing in mobile commerce will give lots of advantage for business with lower cost and high benefits.
\end{abstract}

\section{KEYWORDS}

Mobile Computing, M-Commerce, MCC, E-Commerce, Cloud Computing

\section{INTRODUCTION}

Mobile application is vastly used in this time for various tasks such as shopping, marketing, payment etc. Mobile application faces some challenges like security, low bandwidth, limited storage and processing power hence is not widely used for business perspective. Cloud computing technology provides much large storage capacity, fast computation, security, and the most important on-demand access. Mobile Computing is used for ease of access without having to be connected with a fixed physical link. E-Business provide online platform for enterprises and customers to perform transactions. In globalization era, combination of mobile computing, cloud computing and business intelligence creates new opportunities in market and improves business effectiveness. The limited processing and memory capacity of mobile devices have always needed some use of the cloud for processing of mobile applications and services. The organizations take the advantage of cloud service and mobile application for handling huge data with mobility and security. The benefits of adopting the cloud in the enterprise are discussed in detail and new framework based on the principal is proposed. [1] The paper discuss the two rising technologies i.e. cloud computing and M-Commerce the paper analyse the current actuality of the application for enterprise M-Commerce, and focus on main issues related to that, the impact of the cloud computing upon the E-commerce proves that cloud computing can provide good economic efficiency for the E-commerce application.[2]

SaaS is essentially mobile and couldn't exist without cloud computing infrastructure, [4] can be used anywhere, from any device. SaaS companies, such as Salesforce.com, Concur and workday, provide mobile versions of their different services. The constant migration of major mobile services from devices to back-end cloud servers continues Platform providers such as Apple and Google provides compute and storage on cloud-based platforms.

Mobile Cloud Computing provides benefits such as reduction of costs of information technology and requirement of infrastructure to support the business operation. Mobile Commerce is DOI : 10.5121/ijist.2016.6221 
becoming a reality and more important to all size organizations. This has helped large enterprises to decrease the burden offered by usual information technology services and small and medium enterprises to provide prospective and customized approaches. Small and Medium Enterprises are usually not able to afford large and expensive services and so they can get benefit from MCC that offers online storage, software processing, on-demand access, web browser access, pay per use, interdependency of access device and scalability capacity.

\subsection{Mobile Computing}

Mobile Computing is a technology that allows transmission of data, audio and video through a computer or any other wireless enabled device without having to be connected to a fixed physical channel [12]. Smartphone and tablets are broadly used across all kind of enterprises. Most enterprises are developing applications to support more than one mobile operating system. Mobile application can be developed for all type of mobile operating system. In mobile devices, main two requirements are there for efficient execution of application which is processing power and memory of device. There are two major issues with mobile application is its security of data and limited storage capacity. Now days, the mobile application development require to integrate with cloud computing for better and powerful performance.

\subsection{Cloud Computing}

Cloud computing is collection of remote servers in network to allow centralized data storage and online communication to computer services or resources [10]. Clouds can be categorized as public, private or hybrid. Cloud computing presents when processes and all data are kept on the Internet and not on personal devices to provide on-demand access. Applications are run on a remote server, all the processing task done on remote server and then result sent to the user. Cloud service hosting provides flexibility for on demand access which means user can use the service according to his or her requirements, usually by minutes or hours and also pay per usage.

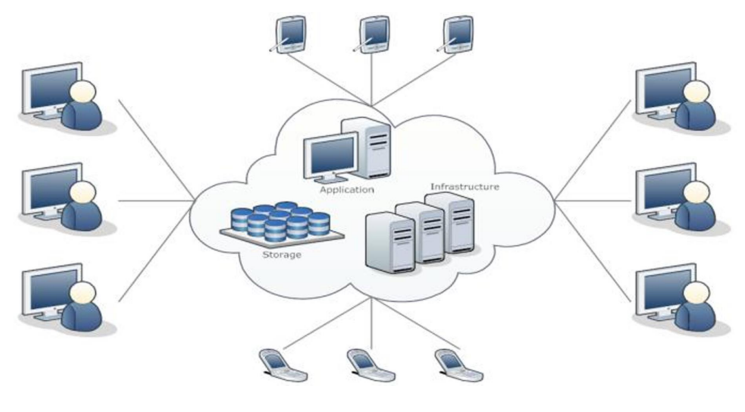

Figure 1. Spam traffic sample

Figure 1 describes Cloud Computing Architecture. Cloud service refers to providing services such as software, infrastructure, and platform to remote customers which are SaaS, IaaS, and PaaS. [6] Big enterprises such as Amazon, Microsoft, Google etc. uses cloud computing in various applications and earn lots of benefits out of it. 


\subsection{Mobile Cloud Computing}

Mobile computing refers to an environment where both the data storage and data processing done on the mobile device. [7] Mobile cloud computing shift the processing power and data storage from the mobile devices into centralized computing platforms placed in clouds and accessed through the wireless connection based. Mobile devices are having many resource challenges like battery life, storage, bandwidth etc. Cloud computing provide facility to user with infrastructure, platforms and software at minimum cost with flexible usage. Mobile Cloud Computing offers mobile users with large storage capacity and high speed processing power without need of high configuration devices as it is grouping of cloud computing, mobile computing and wireless communication. Mobile cloud computing provide many advantages that enhance global business opportunities at lower cost with lots of benefits.

\subsection{Mobile Cloud Services}

There is various cloud service available such as Amazon Web Services that provide service for Infrastructure as a Service (IaaS) and Platform as a Service (PaaS). [6]Many researchers have begun cloud service for mobile cloud computing which enables customization, installation, management of services to be used by the mobile users.

\subsection{E-Business}

E-Business provide platform for the buying and selling of goods and services or the transferring of resources or data over an electronic network using the Internet. These business transactions can be business-to-business, business-to-consumer, consumer-to-consumer or consumer-to-business. [Wiki].E-Business covers all areas starting from product development to product marketing, product selling and online payment. E-Business includes many areas which are E-commerce, EWorking, and E-Procurement-Marketing etc. E-Commerce gives facility for selling online and managing the organization's relationship with the customer. It provides efficiency in organization's internal processes such as online training, budget planning and recruitment process, build up the organization's relationship with its suppliers and consists of product sourcing, purchase management and payment management.

Apart from lots of advantages, E- business is facing some challenges such as cost, security, large data, and interpretability etc. By incorporating Mobile Cloud Computing in E-business, provide great platform for organization and customers.

\subsection{Mobile Commerce}

Mobile Commerce is the E-business on small devise like mobile phones, it is an addition to the Ecommerce in which various [7] E-business activities can be done using small portable hand held devices like mobile phones, tablets etc. at anywhere through wireless technology. [14] The MCommerce and wireless communication technology is being use in E-commerce and give rise to mobile E-commerce in which anyone can find different functionalities such as their locations and do purchase transaction. M-commerce have several issues like low bandwidth, network related problems, low speed etc.

\section{Mobile Cloud Computing In Business}

Business can be more powerful and efficient by incorporating Mobile Computing and Cloud Computing in it. Cloud computing in M-commerce can address various issues and 3G Mobile services provides effectiveness for the mobile related issues, E-commerce development based on cloud computing also solves the problem of scalability and provides on demand services to the 
consumer [3].Many activities such as shopping, mobile balance recharging, ticket booking, billing, recruitment process, advertisement, data sharing can be easily done using MCC.

Mobile Computing

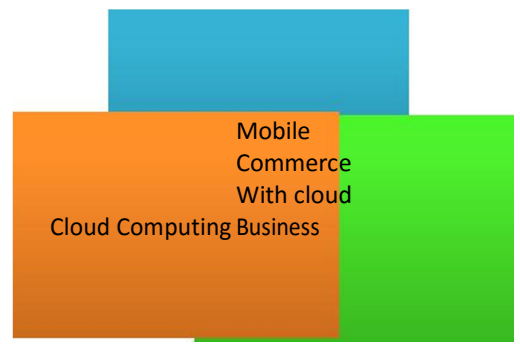

Various algorithms have been introduced to couple m- commerce in cloud architecture i.e. Recommendation algorithm, [3] The system uses mathematical analysis from the past transactional data based on customer mobile number used as the key for the transaction where customers mobile number will be used on every purchase and that key can be used for user rating as well as feedback are taken about the product from in regular intervals. On the server side the mathematical analysis of the product will be done and using recommendation algorithm, accurate recommendation is proposed.

\subsection{Advantages of Mobile Cloud Computing in Business}

\subsubsection{Enterprises will be more cost efficient with mobile cloud computing}

Mobile Cloud Computing allows user to utilize application and resources with minimum investment on software and hardware hence it reduce capital expenditures. Cloud-based mcommerce applications enable business leaders to increase new opportunities without large investments. With smaller capital expenditures needed to maintain application integrated with operational expenditures that are normally payable as you go basis. Organizations can shift their business online with nominal investment risk. A speedy function enables them to restructure time to open market for their products and services with flexibility and scalability at a reasonable investment.

\subsubsection{Consumers have access to more features on their mobile phones}

Mobile cloud computing [8] allow customers to perform online transaction from anywhere at any time with fast access and security.

\subsubsection{Large market can be covered by business with use of mobile cloud}

As Enterprise incorporates [8] mobile cloud computing, it enhances the range of reachability. It useful for small enterprise to large enterprises by on demand and anywhere access that able reach at global market with big amount of customers while investing less revenue.

\subsubsection{MCC provides fast Transaction due to new $(3 \mathrm{~g} / 4 \mathrm{~g})$ technologies}

Every day technologies come up with new and advance features. [11]In Communication technology, due to $3 \mathrm{~g} / 4 \mathrm{~g}$ speed, it enables faster and on the go transaction for users. 


\subsection{Mobile Cloud Computing in Business Issues}

While there are many advantages that of mobile cloud computing, there are some challenges that businesses and customers should be aware of before fully implementing this technology.

\subsubsection{Network unavailability}

The most obvious issue is that you can only access a mobile cloud application when you have access to a working network. Mobile networks will occasionally be unavailable due to various reasons such as atmospheric disturbance.

\subsubsection{Performance}

Users who are in high range from cloud service providers may have experience of high latency and delays in transactions.

\subsubsection{Security}

Enterprises are concerned about security and privacy when using mobile cloud computing. Users are concerned about the vulnerability to attack when information and vital IT resources are not in range of the firewall.

\subsubsection{Control of Business}

Typically, [11] cloud service providers have a full control of the platforms as they do not develop platforms for specific company's environment.

\subsubsection{Bandwidth Costs Cloud computing}

Organization can save money on hardware and software however they could have to pay higher network bandwidth charges as in $\mathrm{m}$-commerce application, it requires large data transactions.

\subsection{Mobile Commerce with Cloud Applications}

\subsubsection{Billing services}

Mobile phone based payment can be done with strong authenticity and security. Mobile Cloud based payment is a service which offers the mobile users and retailers to do transactions more easily as retailers will not require buying any type of devices and the user's device does not require any special configuration hardware to perform the transaction.

\subsubsection{Shopping and Real-time tracking of shipping}

MCC offers Shopping and price comparison over wireless media. User can track their shipment using sensors and satellite based communication systems which are controlled by cloud or network provider.

\subsubsection{Advertisement and alerts}

MCC provides good opportunities for business marketing in various ways such as limited time coupon announcements done by retail stores, big sale advertisement etc. Commodity prices and stock prices alert are provided via notifications and messages. 


\subsubsection{Auction}

Virtual auction can be performed through wireless devices at any location.

\section{CONCLUSION}

Mobile Cloud Computing offers a global and rich environment for business, providing efficiency for small as well as large business with minimum investment and better performance. There are some challenges exist that prevent full fledge implementation of mobile cloud computing in business.

\section{ACKNOWLEDGEMENTS}

I would like to thank everyone.

\section{REFERENCES}

[1] Ahmed AbouElfetouh Saleh (2012) "Proposed Framework based on Cloud Computing for Enhancing Ecommerce Applications”. International Journal of Computer Applications (0975-8887) Volume 59No.5, December 2012.

[2] Chunlin Sun (2012), "Research of E-commerce Based on Cloud Computing". Advances in CSIE, Vol.2 AISC 169 pp 15-20, Springer-verlag Berlin Heidelberg.Denping

[3] Ashfaq Amir Shaikh1, Dr. Gulabchand K. Gupta, "M-COMMERCE RECOMMENDATION WITH MOBILE CLOUD ARCHITECTURE", International Journal of Application or Innovation in Engineering \& Management (IJAIEM) Volume 3, Issue 11, November 2014

[4] http://www.forbes.com/sites/maribellopez/2015/04/06/what-mobile-cloud-means-for-yourbusiness/[accesed on 2 January 2016]

[5] Niroshinie Fernando, Seng W. Loke, Wenny Rahayu, "Mobile cloud computing: A survey" Future Generation Computer Systems 30 May 2012

[6] Yamunadevi.K, Priya.V," Mobile cloud e-business models, services, and applications", Volume: 2 , Issue: 4, 586-590 April 2015

[7] https://en.wikipedia.org/wiki/Mobile_commerce [accessed on 4 January 2016]

[8] http://www.consultparagon.com/blog/5-mobile-cloud-computing-advantages-that-cant-be-ignored

[9] M.Rajendra Prasad, Jayadev Gyani , P.R.K.Murti, "Mobile Cloud Computing: Implications and Challenges", Journal of Information Engineering and Applications Vol 2, No.7, 2012

[10] http://en.wikipedia.org/wiki/Cloud computing [accessed on 2 January 2016]

[11] X. Yang, T. Pan, and J. Shen, "On 3G Mobile E-commerce Platform Based on Cloud Computing," in Proceedings of the 3rd IEEE International Conference on Ubi-Media Computing (U-Media), pp. 198 201, August 2010.

[12] http://en.wikipedia.org/wiki/Mobile_computing [accessed on 3 January 2016]

\section{AUTHORS}

Ms.Nilam S. Desai is assistant professor at smt. chandaben mohanbhai pate institute of computer applications, charotar university of science and technology, changa, gujarat, india. she is master in computer application (mca) from dharmsinh desai university, nadiad, and gujarat, india. her research area is cloud computing, computational biology, data mining techniques, image processing and artificial intelligence. she is having one year and 4 month of teaching experience. 